\title{
Un fardo funerario procedente de Huaca Santa Clara, valle de Virú (ca. 1150 a. D.)
}

Un fardeau funéraire provenant de Huaca Santa Clara dans la vallée de Virú (ca. 1150 A. D.)

A textile funerary bundle from Huaca Santa Clara in the Virú valley (ca. A. D. 1150)

Jean-François Millaire y Flannery Surette

\section{OpenEdition}

\section{Journals}

Edición electrónica

URL: http://journals.openedition.org/bifea/1408

DOI: $10.4000 /$ bifea. 1408

ISSN: 2076-5827

\section{Editor}

Institut Français d'Études Andines

\section{Edición impresa}

Fecha de publicación: 1 agosto 2011

Paginación: 289-305

ISSN: 0303-7495

\section{Referencia electrónica}

Jean-François Millaire y Flannery Surette, « Un fardo funerario procedente de Huaca Santa Clara, valle de Virú (ca. 1150 a. D.) », Bulletin de l'Institut français d'études andines [En línea], 40 (2) | 2011, Publicado el 01 febrero 2012, consultado el 06 noviembre 2020. URL : http://journals.openedition.org/bifea/1408 ; DOI : https://doi.org/10.4000/bifea.1408

\section{(c) $(1) \odot$}

Les contenus du Bulletin de l'Institut français d'études andines sont mis à disposition selon les termes de la licence Creative Commons Attribution - Pas d'Utilisation Commerciale - Pas de Modification 4.0 International. 


\title{
Un fardo funerario procedente de Huaca Santa Clara, valle de Virú (ca. 1150 a. D.)
}

\author{
Jean-François Millaire* \\ Flannery Surette ${ }^{* *}$
}

\begin{abstract}
Resumen
En este artículo se describen los textiles de un fardo funerario descubierto en Huaca Santa Clara, en el valle de Virú, el cual contenía los restos de un niño pequeño acompañado por otros niños y llamas sacrificadas. Los tejidos asociados a este entierro intrusivo — realizado unos cuatro siglos después del abandono del sitio por los miembros de la sociedad Virú - resultan de fundamental importancia dado que no solamente nos ayudan a entender la naturaleza de este complejo evento ritual sino que, además, contribuyen a nuestro conocimiento del arte textil desarrollado durante el periodo Tomaval (Horizonte Medio).
\end{abstract}

Palabras clave: textiles, fardo funerario, ritual mortuorio, valle de Virú, periodo Tomaval

\section{Un fardeau funéraire provenant de Huaca Santa Clara dans la vallée de Virú (ca. 1150 A. D.)}

\section{Résumé}

Cet article décri les textiles d'un fardeau funéraire, découvert sur le site de Huaca Santa Clara dans la vallée de Virú. Il contenait les restes d'un jeune enfant accompagné par d'autres enfants et des lamas

Departamento de Antropología. The University of Western Ontario, London, Ontario, N6A 5C2, Canadá. E-mail: jean-francois.millaire@uwo.ca

** Departamento de Antropología. The University of Western Ontario, London, Ontario, N6A 5C2, Canadá. E-mail: flannerysurette@gmail.com 
ayant fait l'objet d'un sacrifice sacrifiés. Les tissus associés à cet enterrement intrusif —qui eut lieu quelques quatre cents ans après l'abandon du site par les membres de la société Virú- sont d'une grande importance car ils nous aident à mieux comprendre la nature de ce rituel complexe, tout en contribuant à une meilleure connaissance de l'art du tissage durant la période Tomaval (Horizon Moyen).

Mots clés : textiles, fardeau funéraire, rituel mortuaire, vallée de Virú, période Tomaval

\title{
A textile funerary bundle from Huaca Santa Clara in the Virú valley (ca. A. D. 1150)
}

\begin{abstract}
This paper describe the textiles from a funerary bundle from the site of Huaca Santa Clara in the Virú Valley that contained the remains of a young child, accompanied in death by other children and sacrificed Ilamas. The fabrics associated with this intrusive burial event - that took place four hundred years or so after the settlement was abandoned by members of the Viru polity - are of primary importance because they help to document the nature of this complex ritual event, while also adding to our knowledge of Tomaval period textile art of the Middle Horizon.
\end{abstract}

Keywords: textiles, funerary bundle, mortuary ritual, Virú valley, Tomaval period, Middle Horizon

Investigaciones arqueológicas efectuadas recientemente en Huaca Santa Clara, en el valle de Virú (fig. 1), han permitido descubrir los restos asociados a un entierro intrusivo llevado a cabo alrededor del año 1150 d. C., unos cuatrocientos años después del abandono del sitio por los habitantes de la sociedad Virú (Millaire, 2010a; 2010b). Este elaborado evento funerario formó parte de una serie de rituales que incluyeron el enterramiento de niños y crías de llamas en asentamientos abandonados (tanto en el valle de Virú como en el de Moche) durante el Horizonte Medio (Millaire, 2005). Este entierro fue excepcional en términos de su escala y en lo concerniente a la calidad de los restos preservados. El personaje principal fue acompañado a la muerte por otros cinco individuos, así como por llamas sacrificadas, siendo enterrado dentro de un fardo funerario del que destacan sus bien conservados textiles, permitiéndonos visualizar algo del arte textil desarrollado durante este importante periodo histórico de la costa norte. Este artículo se inicia con una explicación del contexto ritual, pasando rápidamente a la descripción de los textiles asociados y a la comparación del fardo funerario con otros ejemplos publicados. La discusión de los datos radiocarbónicos asociados a este contexto ritual llevará a los autores, finalmente, a presentar conclusiones preliminares sobre la naturaleza del evento. 


\section{EL CONTEXTO}

El ritual mencionado anteriormente fue realizado en las derruidas estructuras de Huaca Santa Clara: un sitio encaramado en las laderas de un pequeño cerro del valle de Virú que actualmente domina el pueblo epónimo (figs. 1 y 2). Los resultados del análisis preliminar revelan que el lugar habría funcionado como un centro administrativo Virú durante el periodo Intermedio Temprano, como lo es sugerido por el diseño integral del complejo arquitectónico (instalaciones de almacenamiento a gran escala asociadas a residencias de élite), los patrones de acceso y la naturaleza de los objetos muebles recuperados (Millaire, 2010a). Los miembros de la sociedad Virú abandonaron el asentamiento en algún momento antes del año 760 d. C. 1; sin embargo, este volvió a ser visitado unos cuatrocientos años más tarde para la celebración del ritual mortuorio que se viene estudiando.

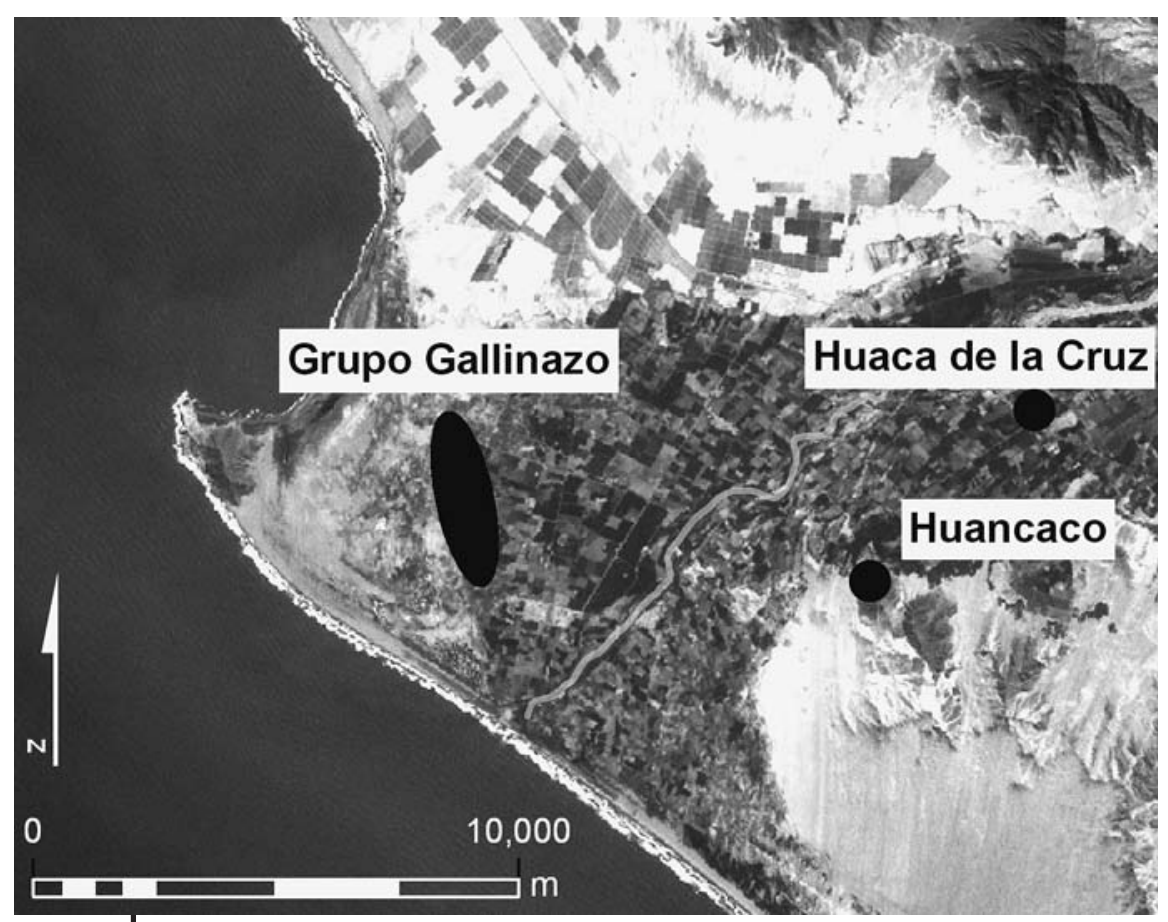

Figura 1 - Imagen satelital del valle de Virú con los principales sitios prehispánicos

NASA Landsat Program 2000, LandsatETM+ sceneELP009R066_7T20000602, SLC-Off, USGS, Sioux Falls

1 Para fechar la ocupación Virú, seis muestras de material orgánico fueron analizadas radiométricamente. Las edades radiocarbónicas convencionales obtenidas estuvieron comprendidas entre los años $2010 \pm 50$ y $1350 \pm 60$ a. p., indicando que el sitio fue ocupado desde aproximadamente el 165 a. C. hasta el $780 \mathrm{~d}$. C. en edades calibradas. Todas las calibraciones presentadas se encuentran basadas en la curva IntCal04 (Reimer et al., 2004) y están expresadas con dos sigmas estadísticos (95 \%). 
El elemento central de este contexto funerario fue un niño de cinco años de edad (entierro 6) que fue depositado al interior de una fosa excavada a través de los niveles de ocupación correspondientes al periodo Virú (figs. 3 y 4). El niño fue enterrado en posición sentada y su cuerpo resultó momificado naturalmente como consecuencia del clima extremadamente seco en esta parte del valle. El individuo llevaba puesto un tocado y había sido cuidadosamente cubierto con una manta y tres paños envoltorios. Una honda larga fue enrollada alrededor del cuello del niño; esta fue posteriormente utilizada para atar el textil que cerró el fardo. A pesar que se creyó inicialmente que el pequeño podría haber sido estrangulado, no hay evidencia física que apoye esta hipótesis (Boston, 2007). Una cría de llama fue enterrada al lado del fardo y cerca de ella se halló un cuenco de mate.

Los restos de cinco individuos más jóvenes también fueron descubiertos en las cercanías aunque en fosas menos profundas. El primero de ellos (entierro 4) correspondió a un subadulto de aproximadamente 13 ó 14 años enterrado en posición flexionada, recostado sobre su lado derecho; el cuerpo presentaba un mal estado de conservación debido a su proximidad con la superficie. Los restos de un individuo más joven (4 años) fueron hallados a menos de un metro

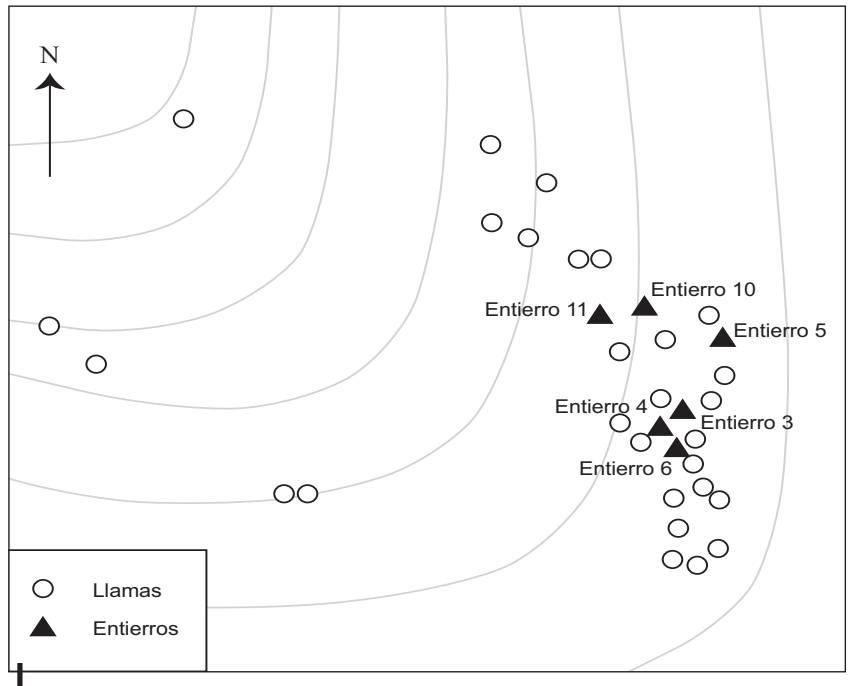

Figura 3 - Ubicación de los entierros y llamas de Huaca Santa Clara de distancia (entierro 3); se encontraba tendido de espaldas sobre una cría de llama (también depositada de espaldas). El análisis de su esqueleto reveló que había sido mutilado antes o inmediatamente después de su muerte: marcas de cortes fueron identificadas en sus costillas derechas y en su esternón; un patrón que sugiere fuertemente la realización de prácticas de sacrificio mediante cortes en la garganta (Boston, 2007). Hacia el norte, un tercer niño de entre 9 y 10 años (entierro 11) fue enterrado de espaldas con su mano derecha colocada sobre la cabeza. Tres tejidos llanos fueron 


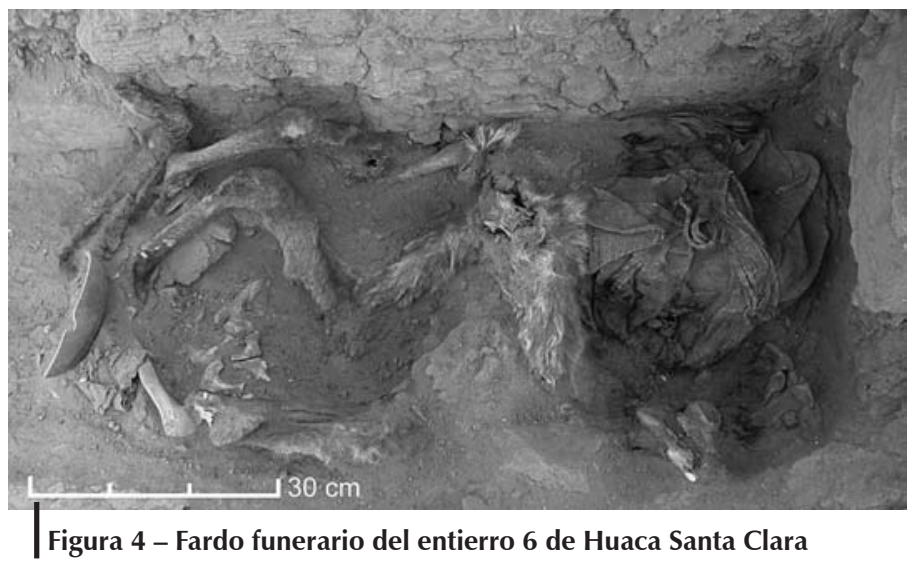

encontrados cerca del esqueleto. Los cuerpos de otros dos individuos fueron descubiertos en las cercanías. El primero (entierro 5) pertenecía a un niño de entre 7 y 8 años que había sido enterrado recostado sobre su lado izquierdo cerca a otra cría de llama. El segundo (entierro 10) correspondió a un niño de entre 8 y 9 años enterrado boca abajo. Dos tejidos llanos estuvieron asociados a este individuo. Marcas de cortes fueron identificadas en su tercera, cuarta y quinta costilla del lado izquierdo así como en su esternón (Boston, 2007). Las evidencias expuestas sugieren que al menos dos de estos niños fueron sacrificados como parte del ritual funerario.

Durante el evento, un total de veintiocho llamas fueron sacrificadas y enterradas cerca de los niños (figs. 3, 4 y 5). Los animales fueron colocados en diferentes posiciones: algunos se presentaban enrollados, mientras que otros yacían de espaldas o boca arriba. Unos cuantos fueron colocados en posiciones contorsionadas; sin embargo, sugerimos que ellos habrían sido «arrojados» al interior de las fosas en vez de ser cuidadosamente depositados. Estos animales probablemente fueron sacrificados con un instrumento punzocortante, tal como lo evidencian las marcas de cortes sobre las costillas de al menos dos de las Ilamas. Los animales pudieron haber sido atados antes de ser extendidos, tal como lo indica la presencia de una cuerda enroscada alrededor de las patas delanteras y traseras de por lo menos uno de los animales. Todas las llamas estuvieron extremadamente bien conservadas; en muchos casos la piel, el pelo y los órganos internos se mantuvieron casi intactos. Un análisis de los esqueletos de las Ilamas indica que sólo un segmento específico del total de su población fue seleccionado para el ritual. Veintiún llamas tenían menos de tres meses cuando fueron sacrificadas, mientras que otras cuatro tenían entre tres y seis meses de edad2.Las tres restantes también eran crías, pero fue imposible determinar su edad con mayor exactitud. 


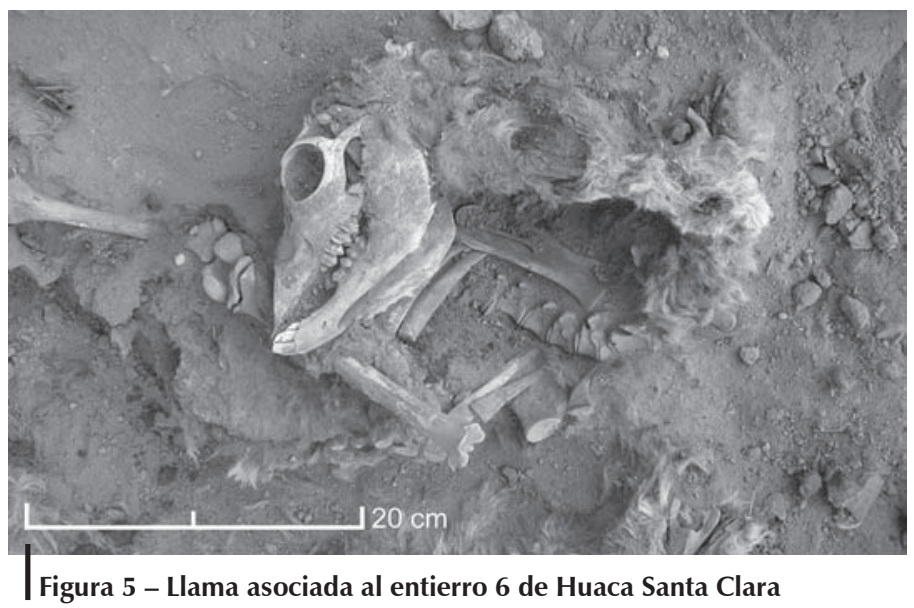

Finalmente, en esta área fue encontrada una ofrenda que contenía las valvas de seis conchas de Spondylus (Spondylus sp.) —un tipo de material ampliamente utilizado en la costa norte prehispánica durante los periodos tardíos - rodeadas por adobes disueltos. En sus cercanías también fue hallado un textil enfardelado y decorado con plumas.

Este evento fue probablemente parte de una práctica ritual más amplia documentada en otros asentamientos del valle de Virú y en varios sitios a lo largo de la costa (Millaire, 2005). Rituales similares han sido identificados, por ejemplo, en Huancaco (Bourget, 2010), Huaca Negra (Strong \& Evans, 1952) y Cerro de Huarpe (Willey, 1953) en Virú; las Huacas de Moche (Uhle, 1899; Steve Bourget, comunicación personal, 2005) y Huanchaco (Donnan \& Foote, 1978) en el valle de Moche, e incluso en los valles sureños de Chao (Kent et al., 2009) y Nepeña (David Chicoine, comunicación personal, 2006). Sin embargo, el entierro de Huaca Santa Clara resulta especial por la calidad de su conservación, realmente sobresaliente para los estándares de la costa norte. Esto se hizo especialmente obvio cuando se analizaron los textiles recuperados.

\section{LOS TEXTILES DEL FARDO 3}

El fardo fue asegurado con una honda hecha de fibra vegetal y contiene cinco textiles, dos de los cuales tienen decoración. Uno presenta diseño de listas de colores en dirección de la urdimbre. El otro representa un complejo diseño de perfil escalonado, ejecutado con una trama suplementaria (brocado) de algodón color marrón oscuro.

3 Análisis textil basado en los estudios de d'Harcourt (2002 [1962]), Emery (1995 [1966]) y SeilerBaldinger (1979). 


\section{1. HSC 183}

Esta pieza es identificada como un posible tocado aunque su conservación no es buena. Fue tejida en técnica 2/1 (urdimbres dobles y tramas solas de algodón marrón) con hilos de un solo cabo hilado en S. El ancho es de aproximadamente $20 \mathrm{~cm}$, mientras que el largo es de $80 \mathrm{~cm}$.

\section{2. HSC 184}

Este tejido decorado (fig. 6) fue encontrado alrededor del cuerpo momificado, mide aproximadamente $100 \mathrm{~cm}$ por $135 \mathrm{~cm}$. Está constituido por dos piezas cosidas conjuntamente, con dos de las esquinas atadas entre sí. Se sugiere que fue una manta. Presenta urdimbres de diferentes colores de algodón natural y teñido, fueron montadas en el telar y luego tejidas con una trama de algodón de color natural marrón claro. Todos los hilos son

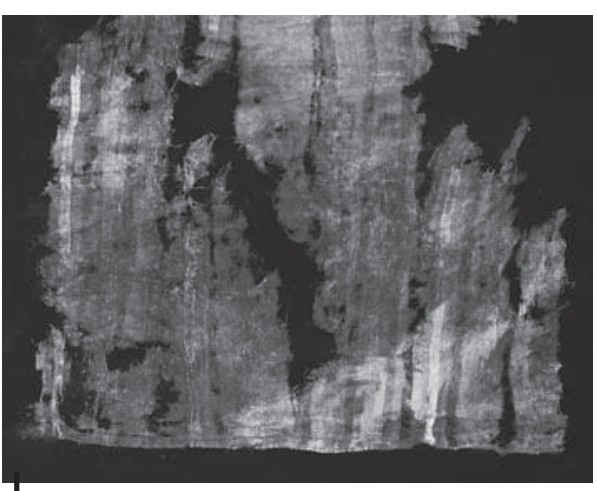

Figura 6 - Textil HSC 184 de Huaca Santa Clara de un solo cabo hilado en S. Las rayas de colores dominan el diseño de la vestimenta (fig. 7): el número de urdimbres por centímetro es de 20, mientras que la trama es de 9 por centímetro. La mayoría de las rayas angostas están tejidas en 1/1 (urdimbres y tramas solas), mientras que la pieza central de algodón natural y las rayas marrón chocolate fueron tejidas en 2/1 (con urdimbres pareadas o dobles).

Los colores originales presentes en las listas de urdimbre son difíciles de determinar debido a que el tejido fue manchado por la descomposición del cuerpo; sin embargo, tentativamente podemos precisar los siguientes colores: marrón claro, marrón oscuro, verde, crema, azul y chocolate. El azul pudo ser añil (Indigofera suffruticosa) o llangua (Cybistax antisyphilitica), un tinte insoluble en el agua (Antúnez de Mayolo, 1989: 183). Los demás tonos (marrón claro, marrón oscuro y crema) están presentes de manera natural en el algodón nativo peruano, no obstante, los marrones oscuros podrían haber sido obtenidos a partir de tintes compuestos por taninos (Wouter \& Rosario-Chirinos, 1992: 241).

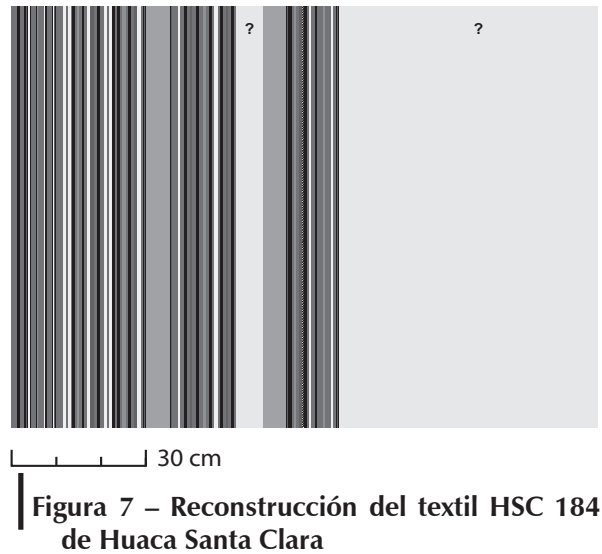
de Huaca Santa Clara 


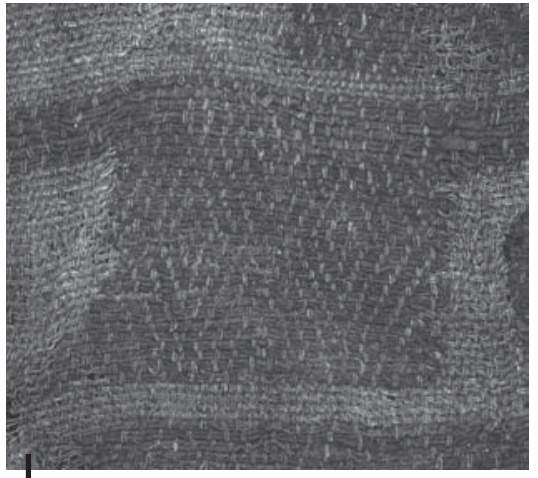

Figura 8 - Detalle del textil HSC 186 de Huaca Santa Clara

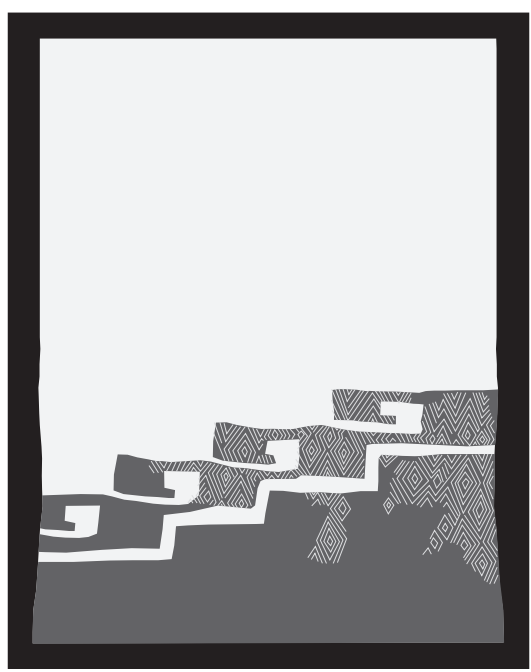

Figura 9 - Reconstrucción del textil HSC 186 de Huaca Santa Clara

\section{3. HSC 185}

Éste es el primero de los tres paños envoltorios. Es una prenda larga hecha de dos piezas tejidas en tela llana cosidas juntas. La medida del primer panel es $170 \mathrm{~cm}$ por $53 \mathrm{~cm}$. Fue ejecutado con urdimbres y tramas dobles de algodón crema, hilado en $\mathrm{S}$. Restos de ambos orillos están presentes: un orillo simple al borde y un orillo de dos cordones de ocho hilos para la trama. Los cordones pasan a través de la tela horizontalmente y forman picos gruesos. El segundo panel es del mismo ancho, aunque de $10 \mathrm{~cm}$ menos de largo.

\section{4. HSC 186}

El segundo paño envoltorio es una ancha pieza textil $(360 \mathrm{~cm}$ por $65 \mathrm{~cm})$ de algodón crema, tejida en 2/2 (urdimbres y tramas dobles) con hilos de un solo cabo hilados en S. Un extremo presenta un área con diseños de escalones y olas ejecutados en técnica de brocado a partir de dos tramas suplementarias de algodón marrón oscuro, que en su paso bajo las urdimbres pareadas configuran rombos concéntricos o de un patrón de diamante (figs. 8 y 9)4. Este motivo en brocado utiliza tramas dobles que van de borde a borde de la trama en el sector inferior del área con motivo escalonado (brocado total); para la realización del motivo de olas, es visible que en los bordes de cada forma principal los hilos de la trama suplementaria retornan para completar la hilera siguiente (brocado parcial). Este tipo de decoración con trama suplementaria en diseño de diamante es bastante similar a un ejemplo descubierto en Pacatnamú (Hecker \& Hecker, 1995: 156-157).

\section{5. HSC 187}

Éste es el tercer paño envoltorio asociado a este fardo. Fue elaborado de dos piezas de tela llana 2/2 (urdimbres y tramas dobles) en algodón marrón claro,

4 La trama suplementaria es ejecutada usando tramas dobles que pasan sobre dos series de urdimbres dobles y debajo una serie de urdimbres dobles, ambos rellenos en la forma pero además creando un patrón de diamante. 
de un solo cabo hilado en S, de $175 \mathrm{~cm}$ por $60 \mathrm{~cm}$. El borde de la trama fue asegurado con tres cordones de doce hilos. Los cordones pasan a través de la tela horizontalmente y forman picos gruesos.

\section{OTROS TEXTILES DEL CONTEXTO FUNERARIO}

Varios textiles fueron recuperados en las cercanías de los cuerpos de los niños, incluyendo uno recubierto de plumas.

\section{1. HSC 275a}

El entierro 10 contenía dos piezas diferentes. La primera fue identificada como un posible tocado basado en sus dimensiones y en su posición cercana al cráneo. Fue tejido en tela llana 2/2 (urdimbres y tramas dobles) con algodón crema hilado en S. El ancho total es aproximadamente de $26 \mathrm{~cm}$, mientras que el largo es de $39 \mathrm{~cm}$.

\section{2. HSC 275b}

Un segundo textil fue encontrado con el entierro 10. Fue identificado como un posible taparrabo, debido a sus dimensiones y a su posición cercana a la pelvis. El textil fue tejido en tela llana 2/2 (urdimbres y tramas dobles) con hilos de algodón color crema hilados en $\mathrm{S}$; tiene un ancho de $5 \mathrm{~cm}$, mientras que el largo es de $120 \mathrm{~cm}$.

\section{3. HSC 251a}

El entierro 11 contenía tres piezas distintas. La primera fue identificada como un posible tocado, debido a sus dimensiones y a su posición cercana al cráneo. Fue tejida en tela llana 2/2 (urdimbres y tramas dobles) con hilos de algodón color crema hilados en S. El ancho es aproximadamente $16 \mathrm{~cm}$, mientras que el largo es de $34 \mathrm{~cm}$.

\section{4. HSC 251c}

El segundo textil asociado con el entierro 11 fue identificado como un posible taparrabo, basado en sus dimensiones y en su posición cercana a la pelvis. Fue tejido en tela llana 2/2 (urdimbres y tramas dobles) con hilos de algodón color crema hilados en S. El ancho es de aproximadamente $13 \mathrm{~cm}$, mientras que el largo sobrante es de $300 \mathrm{~cm}$. 


\section{5. HSC 251d}

La tercera pieza descubierta en el entierro 11 fue identificada como un posible paño envoltorio, debido a sus dimensiones y a su posición debajo del esqueleto. Consiste en una larga pieza creada a partir de dos piezas tejidas individualmente, pero cosidas juntas. El fragmento conservado mide $60 \mathrm{~cm}$ por $66 \mathrm{~cm}$ y fue ejecutado en tela llana 2/1 (urdimbres dobles y tramas solas) con hilos de algodón crema hilado en $\mathrm{S}$.

\section{6. HSC 272}

Un textil con plumas fue descubierto próximo a la actual superficie del sitio, cerca del escondrijo de conchas de Spondylus. La base del vestido es un tejido llano de algodón crema hilado en $\mathrm{S}$ en técnica 2/1 (urdimbres dobles y tramas solas) y sus medidas son $138 \mathrm{~cm}$ por $51 \mathrm{~cm}$. Están presentes sus cuatro orillos (orillos simples a los bordes y un orillo de cordones de seis hilos para la trama. Los cordones pasan a

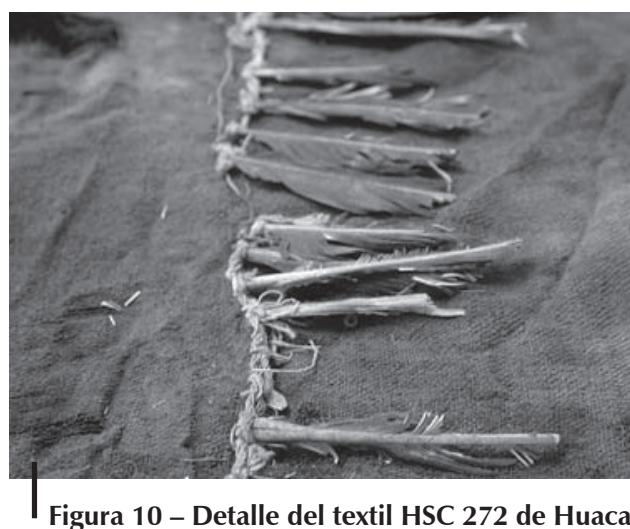
Santa Clara

través de la tela horizontalmente y forman picos gruesos). Los orillos superiores e inferiores están doblados. Este textil está decorado con hileras de plumas iridiscentes azul y verde (fig. 10). El tejido tuvo originalmente como mínimo ocho hileras con aproximadamente 40 plumas. Los cálamos de las plumas están doblados sobre una cuerda gruesa y de hilos múltiples de algodón crema; una segunda cuerda alrededor de dichos cálamos aseguran las plumas (d'Harcourt, 2002 [1962]: 132). A diferencia de la ilustración que presenta Raoul d'Harcourt, la primera cuerda también pasa debajo de la segunda. En cuanto a las especies, estas plumas pudieron haber provenido de una variedad de loros amazónicos grandes: el guacamayo azul y amarillo (Ara ararauna) o el guacamayo rojo (Ara chloroptera). Un textil similar fue descubierto en Zapallán $(27,4 \mathrm{~km}$ al norte de Lima) en asociación con el estilo de los fardos funerarios Chancay (Lothrop \& Mahler, 1978 [1957])5.

5 Aunque fue hallado fuera de contexto, un segundo textil con plumas (HSC 208b) pudo originalmente haber sido llevado al cerro durante el evento ritual. La tela base está compuesta de algodón crema hilado uniformemente en S con urdimbres y tramas dobles. Se ha logrado preservar el total de su ancho $(66 \mathrm{~cm})$ mientras que el largo aún conservado es de $24 \mathrm{~cm}$. Había sido decorado con tres bandas monócromas de pequeñas plumas grises y blancas. Éstas habían sido aseguradas utilizando cuerdas de algodón hilado y torcido densamente, de coloración natural crema y marrón claro. Primero el raquis de la pluma era doblado y una densa cuerda de algodón hilada en S era pasada a través de él (véase Rowe, 1984: 154). Luego dos cuerdas de algodón hiladas en S y torcidas en Z eran anudadas consecutivamente debajo del raquis de la pluma, asegurándolos a la tela que servía de soporte. Estas hileras de plumas eran colocadas muy juntas hasta cubrir la tela por completo. Debido al color y el tamaño, es difícil identificar categóricamente las especies de aves a las que pertenecían 


\section{DISCUSIÓN}

Basándonos en la presencia de tejidos elaborados con tela llana en ligamento 2/1 y $2 / 2$ con hilos de algodón de un solo cabo torcidos hacia la izquierda, en S, para realizar paños con dos orillos simples y dos orillos con cordones gruesos, se puede concluir que las piezas bajo estudio definitivamente fueron confeccionadas en el litoral sin influencia de la sierra. Es más, estos tejidos Ilanos mantienen el patrón característico de los tejidos norteños que en cuanto a ligamento difieren de los de la costa central (Z2S), pues los tejidos llanos del litoral norte son fabricados con hilos de un solo cabo torcidos en S, con rangos cromáticos a partir de colores naturales y teñidos en algodón que proporcionan tonos apastelados o mates. A ello se añade la presencia de textiles con plumas y fibras de camélido. Por todo esto se concluye que los textiles de este entierro son similares a los del periodo Chimú Tardío.

Este entierro es importante debido a que solo unos cuantos fardos funerarios similares han sido reportados y analizados en Virú, en los sitios de Huaca de la Cruz, Huaca Gallinazo y en Castillo de Tomaval (Bennett, 1939; Strong \& Evans, 1952). Además, hay solo unos cuantos ejemplares comparables procedentes de la costa norte. Un grupo de fardos funerarios recientemente recuperados de la fachada de la Huaca Cao Viejo, en el valle de Chicama, proporciona algunas bases para la comparación (Fernández, 2001; Franco \& Gálvez, 2005; Mujíca, 2007). En un fardo del periodo Transicional procedente de este último sitio, por ejemplo, fueron hallados los restos de un hombre de entre 35 y 40 años de edad que había sido enterrado acompañado de una mujer sacrificada y de un infante, además de varias vasijas de cerámica. Este fardo contenía más capas que el ejemplo de Huaca Santa Clara, incluyendo camisetas con mangas cuyos cuellos, costados y mangas se encontraban decoradas con diseños geométricos y de seres antropomorfos de estilo Huari representados sobre tapices confeccionados con fibra de camélido (Franco \& Gálvez, 2005). Entierros elaborados que datan del subsiguiente periodo Lambayeque Temprano también fueron descubiertos; estos poseían fardos textiles con falsas cabezas (con máscaras) y extremidades. Más al norte, en Pacatnamú, un número de fardos funerarios del periodo Intermedio Tardío fueron encontrados por Heinrich Ubbelohde-Doering a inicios del siglo XX (Hecker \& Hecker, 1995), pero desafortunadamente muy poca información útil ha sido publicada. Estos fardos —al igual que otros (véase Conrad, 1974; Donnan \& Mackey, 1978; Rowe, 1984; Vreeland, 1998) — parecen derivar de una tradición funeraria parecida a la que se documentó en Huaca Santa Clara, pero más allá de las comparaciones, se espera las publicaciones completas sobre estos importantes hallazgos arqueológicos.

estas plumas, particularmente en el caso de las de color blanco. El pato criollo (Cairina moschata) es un posible candidato, dado que en su versión domesticada puede presentar variedades de colores que incluyen el blanco y el gris. Sin embargo, las plumas blancas también pudieron haber sido obtenidas de especies costeñas tales como la garza blanca (Egretta alba), la garceta nívea (Egretta thula), el cormorán guanay (Phalacrorax bougainvillii) y el pato crestudo (Sarkidiornis melanotos sylvicola) (O'Neill, 1984). 
Los fardos funerarios son mucho más abundantes en la costa central (véase Córdova, 2005; Fernandini, 2006; Fleming, 1987; Fleming et al., 1983; Frame et al., 2004; Kaulicke, 1997; Lothrop \& Mahler, 1978 [1957]; Squier, 1967 [1869]; Stothert, 1978; 1979; Vreeland, 1998; Williams, 2005). Por ejemplo, cerca de 1800 fardos funerarios fueron recuperados cerca de la actual localidad de Ancón entre 1945 y 1962 (Stothert, 1979), a pesar que solo pocas descripciones y análisis han sido publicados y éstos tienden a presentar los ejemplos más espectaculares. Estos fardos casi siempre fueron construidos con muchas capas de textiles (incluyendo falsas cabezas) y copos de algodón sin procesar o materiales vegetales; no obstante, algunos también fueron enterrados con una sola capa de textiles (Fernandini, 2006). Una situación similar ha sido observada en la excavación efectuada recientemente en el cementerio del Horizonte Tardío de Puruchuco-Huaquerones, caracterizada por el hallazgo de un gran número de cuerpos momificados envueltos en fardos funerarios (Williams, 2005). A lo largo de la costa sur peruana, la costumbre de enterrar a los muertos en fardos funerarios ha sido incluso mejor estudiada $y$, gracias al clima extremadamente seco, varias generaciones de investigadores han sido capaces de documentar esta tradición a través del tiempo (Dwyer, 1979; Dwyer \& Dwyer, 1975; Kroeber \& Collier, 1998; Paul, 1982; 1990; Peters, 1991; 1997; 2000; Tello \& Mejía, 1979; Yacovleff \& Muelle, 1934).

Las comparaciones entre el ritual de Huaca Santa Clara y los contextos arqueológicos registrados más allá de Virú inevitablemente implican hacer cotejos a grandes distancias; no obstante, esta breve revisión ayuda a poner el ritual estudiado en perspectiva. Cuando se compara con hallazgos similares descubiertos más al norte o al sur, este fardo funerario parece bastante modesto, habiendo requerido solamente una pequeña cantidad de textiles. En efecto, el total de tela necesitada incluyó tan sólo cerca de $10 \mathrm{~m}^{2}$, cantidad relativamente pequeña considerando el abundante hilo de algodón utilizado en algunos fardos funerarios de otros sitios localizados a lo largo de la costa. Por ejemplo, James Vreeland describe un fardo proveniente de la costa central que tuvo un estimado de $265 \mathrm{~km}$ de hilo de un doblez, el cual debió haber requerido alrededor de cuatro mil horas de hilado y doblado (Vreeland, 1998).

Lo expuesto demuestra que el cálculo del tiempo empleado para producir incluso una pequeña cantidad de prendas es importante, y basándonos en el estudio de Karen Stothert en un contexto funerario de Ancón del Horizonte Tardío (1978: 18), se estima que la producción de la tela necesaria para el ritual estudiado habría tomado alrededor de 600 horas. Si a ello se suma el hecho de que algunas de las telas fueron decoradas y el considerable consumo de energía invertido en este evento (sacrificios humanos y de llamas, bienes importados como plumas amazónicas y conchas de Spondylus procedentes del litoral ecuatoriano), resulta seguro sostener que el ritual involucró una considerable dedicación de trabajo y recursos de la comunidad, lo que sugiere que el estatus de este pequeño niño justificaba un tratamiento especial tras su muerte.

Exceptuando los textiles estudiados, ningún otro artefacto culturalmente diagnóstico fue hallado en asociación con los entierros. La información contextual 
(Millaire, 2005; 2010a) indica que ellos representan un evento intrusivo tardío; no obstante, este tuvo lugar en lo que ya era para ellos un asentamiento en ruinas. El fechar este acontecimiento fue por consiguiente fundamental, dos muestras del material orgánico fueron analizadas utilizando la técnica de fechado radiocarbónico estándar. El primer fechado (Beta-186962) fue obtenido de una pequeña porción de algodón procedente del fardo funerario. La muestra arrojó una edad radiocarbónica de $780 \pm 60$ a. p. (1150 a 1300 d. C. en edades calibradas). La segunda muestra (Beta-195616) provino del fémur de una de las llamas (llama 21), y arrojó una edad radiocarbónica de $980 \pm 40$ a. p. (990 a 1160 d. C. en edades calibradas). Si ambas fechas son correctas, sugerirían que el ritual tuvo lugar cerca del año $1150 \mathrm{~d}$. C.

La segunda mitad del siglo XII fue un tiempo de cambio en Virú, marcado por la expansión Chimú hacia el sur de su capital (Topic, 1990; Mackey \& Klymyshyn, 1990). Sin embargo, aún se desconoce si la región estuvo bajo el control de Chan Chan cuando el ritual fue Ilevado a cabo. Aunque los chimúes tomaron eventualmente el control del valle, ninguna de sus típicas cerámicas de pasta negra impresas en molde ha sido encontrada en Huaca Santa Clara, menos aún en asociación con estos entierros. Por consiguiente, se puede señalar con seguridad que este elaborado tratamiento mortuorio no fue realizado por agentes chimúes, sino por grupos pertenecientes a un movimiento religioso local. En Virú, el periodo previo a la presencia Chimú es conocido como Tomaval: un tiempo asociado con importantes cambios culturales marcados por influencias desde la sierra y la costa central, particularmente visible en la arquitectura cívica, el arte cerámico y las prácticas rituales (Ford, 1949; Millaire, 2002; Willey, 1953). Este incremento del contacto con sociedades distantes pudo también haber estimulado cambios en el arte textil; sin embargo, muy pocos textiles con documentación contextual son conocidos para esta fase de la región.

Durante este periodo, una de las principales transformaciones fue el cambio en la posición de los entierros, del tradicional cuerpo extendido se pasó al flexionado sentado (Conrad, 1982; Donnan \& Cock, 1986; Donnan \& Mackey, 1978; Hecker \& Hecker, 1995; Heyerdahl et al., 1995; Isbell, 1997; Mackey, 1982; Millaire, 2002; 2005; Moseley, 1982; 2001). Esto se vio obviamente acompañado por un cambio en el envolvimiento del cadáver, que hasta entonces se había realizado esencialmente con paños envoltorios y al interior de ataúdes o cajas reforzadas con cañas y ramas (Donnan, 1995; Millaire, 2002). La nueva forma de entierro, mantenida hasta la conquista española (Rowe, 1946; 1995), constituye un complejo fenómeno que probablemente refleje un gran cambio en las creencias concernientes a los propios ritos de pasaje. El evento ritual documentado en Huaca Santa Clara podría muy bien representar un contexto temprano en el cual este nuevo tratamiento funerario fue utilizado. Si bien en tiempos posteriores todas las personas fallecidas eran enterradas en posición flexionada sentada (cualquiera fuera su posición social), es digno de mencionar que en este caso solamente el personaje principal (un niño de 5 años) fue «complacido» con este nuevo tratamiento (todos los demás presentaron posición extendida). ¿Quién fue este niño?, ¿en mérito a qué tuvo este elaborado 
funeral?, o quizás debiéramos preguntarnos ìhijo de quién era este niño? (Gillespie, 2001: 77). Claramente se ha perdido una parte importante de esta historia; sin embargo, la información procedente de los textiles asociados al evento que aquí se ha presentado aportan algo a nuestra comprensión de este complejo ritual y a nuestro incipiente conocimiento sobre el arte textil del periodo Tomaval.

\section{Agradecimientos}

Los autores quisieran agradecer a Magali Morlion por la meticulosa limpieza, catalogación y análisis inicial de los textiles encontrados en Huaca Santa Clara. Si no hubiera sido por su dedicación mucho se hubiera perdido y nosotros, además, no hubiéramos tenido la maravillosa oportunidad de conocer más de cerca a los antiguos pobladores de Virú a través de su extraordinario arte textil. El presente trabajo fue apoyado por el Fonds de Recherche sur la Société et la culture de Québec, por el Social Sciences and Humanities Research Council of Canada, por la Dumbarton Oaks Research Library and Collection y por The University of Western Ontario. Finalmente agradecemos a Arabel Fernández y a Carmen Thays por sus consejos durante el análisis textil así como a Magali Morlion y Sergio Barraza por su inestimable ayuda en preparar la versión final de este texto.

\section{Referencias citadas}

ALTAMIRANO, A., 1987 - Desarrollo de los camélidos sudamericanos. Boletín de Lima, 49: 9-16.

ANTÚNEZ DE MAYOLO, K. K., 1989 - Peruvian Natural Dye Plants. Economic Botany, 43 (2): 181-191.

BENNETT, W. C., 1939 - Archaeology of the North Coast of Peru: An Account of Exploration and Excavation in Viru and Lambayeque Valleys, 153 pp.; New York: American Museum of Natural History.

BOSTON, C. E., 2007 - Skeletal Inventory of Huaca Santa Clara Collection; London, Ontario: Virú Polity Project. Manuscript on file.

BOURGET, S., 2010 - Cultural Affiliation during the Early Intermediate Period: The Huancaco Case, Virú Valley. In: New Perspectives on Moche Political Organization (J. Quilter \& L. J. Castillo, eds.): 201-222; Washington, D. C.: Dumbarton Oaks Research Library \& Collection.

CONRAD, G., 1974 - Burial Platforms and Related Structures on the North Coast of Peru: Some Social and Political Implications; Cambridge, Massachusetts: Harvard University. PhD dissertation.

CONRAD, G., 1982 - The Burial Platforms of Chan Chan: Some Social and Political Implications. In: Chan Chan: Andean Desert City (M. E. Moseley \& K. C. Day, eds.): 87-117; Albuquerque: University of New Mexico Press.

CÓRDOVA, M., 2005 - El Sauce, un cementerio del Horizonte Tardío en San Juan de Lurigancho, Lima. Corriente Arqueológica, 1: 199-221.

D'HARCOURT, R., 2002 [1962] - Textiles of Ancient Peru and their Techniques, 320 pp.; New York: Dover Publication. 
DONNAN, C. B., 1995 - Moche Funerary Practice. Tombs for the Living: Andean Mortuary Practices (T. D. Dillehay, ed.): 111-159; Washington, D. C.: Dumbarton Oaks Research Library and Collection.

DONNAN, C. B. \& COCK G. (eds.), 1986 - The Pacatnamu Papers, volume 1, 188 pp.; Los Angeles: Museum of Cultural History, University of California.

DONNAN, C. B. \& FOOTE, L. J., 1978 - Appendix 2. Child and Llama Burials from Huanchaco. In: Ancient Burial Patterns of the Moche Valley, Peru (Ch. B. Donnan \& C. J. Mackey): 399-408; Austin: University of Texas Press.

DONNAN, C. B. \& MACKEY, C. J., 1978 - Ancient Burial Patterns of the Moche Valley, Peru, 412 pp.; Austin: University of Texas Press.

DWYER, J. P., 1979 - The Chronology and Iconography of Paracas-Style Textiles. In: The Junius B. Bird Pre-Columbian Textile Conference (A. P. Rowe, E. P. Benson \& A.-L. Schaffer eds.): 105-128; Washington D. C.: The Textile Museum \& Dumbarton Oaks.

DWYER, E. B. \& DWYER, J. P., 1975 - The Paracas Cemeteries: Mortuary Patterns in a Peruvian South Coastal Tradition. In: Death and the Afterlife in Pre-Columbian America (E. P. Benson, ed.): 145-161; Washington, D. C.: Dumbarton Oaks Research Library Collections.

EMERY, I., 1995 [1966] - The Primary Structures of Fabrics: An Illustrated Classification, 341 pp.; Washington, D. C.: The Textile Museum.

FERNANDINI, F. G., 2006 - Contextos funerarios del Horizonte Medio en la Necrópolis de Ancón. Revista Electrónica de Arqueología PUCP, 1: 23-51.

FERNÁNDEZ, A., 2001 - Indumentaria elaborada en paneles. Revista Arqueológica SIAN, 11: 26-33.

FLEMING, S., 1987 - Infant Sacrifice at Pachacamac, Peru: Dignity in Death. Archaeology, 40: 64-65.

FLEMING, S., MULLER, W. T. \& BRAHN J. L., 1983 - The Mummies of Pachacamac. MASCA, 2: 139-156.

FORD, J. A., 1949 - Cultural Dating of Prehistoric Sites in Virú Valley, Peru. In: Surface Survey of the Virú Valley, Peru (J. A. Ford \& G. R. Willey): 29-87; New York: American Museum of Natural History.

FRAME, M., GUERRERRO, D., VEGA, M. \& LANDA, P., 2004 - Un fardo funerario del Horizonte Tardío del sitio Rinconada Alta, valle del Rímac. Bulletin de I'Institut Français d'Études Andines, 33: 815-860.

FRANCO, R. \& GÁLVEZ, C., 2005 - Muerte, identidades y prácticas funerales postmochicas en el complejo el Brujo, Valle de Chicama, costa norte del Perú. Corriente Arqueológica, 1: 70-118.

GILLESPIE, S. D., 2001 - Personhood, Agency, and Mortuary Ritual: A Case Study from the Ancient Maya. Journal of Anthropological Archaeology, 20: 73-112.

HECKER, G. \& HECKER, W., 1995 - Die Grabungen von Heinrich Ubbelohde-Doering in Pacatnamu, Nordperú: Untersuchungen zu den Huacas 31 und 14 sowie Bestattungen und Fundobjekte; Berlin: D. Reimer.

HEYERDAHL, T., SANDWEISS D. H. \& NARVÁEZ, A., 1995 - Pyramids of Túcume: The Quest for Peru's Forgotten City, 240 pp.; London: Thames and Hudson.

ISBELL, W. H., 1997 - Mummies and Mortuary Monuments: A Postprocessual Prehistory of Central Andean Social Organization, 371 pp.; Austin: University of Texas Press.

KAULICKE, P., 1997 - Contextos funerarios de Ancón, 123 pp.; Lima: Fondo Editorial PUCP. 
KENT, J. D., ROSALES, T., VÁSQUEZ, V., BUSCH, R. A. \& GAITHER, C. M., 2009 - Gallinazo and Moche at the Santa Rita B Archaeological Complex, Middle Chao Valley. In: Gallinazo: An Early Cultural Tradition on the Peruvian North Coast (J.-F. Millaire \& M. Morlion, eds.): 167-179; Los Angeles: Cotsen Institute of Archaeology Press.

KROEBER, A. L. \& COLLIER, D., 1998 - The Archaeology and Pottery of Nazca, Peru: Alfred L. Kroeber's 1926 Expedition (P. H. Carmichael, ed.), 283 pp.; Walnut Creek, California: AltaMira Press.

LOTHROP, S. K. \& MAHLER, J., 1978 [1957] - A Chancay-Style Grave at Zapallan, Peru: An Analysis of its Textiles, Pottery and Other Furnishings, 38 pp.; Cambridge, Massachusetts: Peabody Museum.

MACKEY, C. J., 1982 - The Middle Horizon as Viewed from the Moche Valley. In: Chan Chan: Andean Desert City (M. E. Moseley \& K. C. Day, eds.): 321-331; Albuquerque: University of New Mexico Press.

MACKEY, C. J. \& KLYMYSHYN, A. M. U., 1990 - The Southern Frontier of the Chimu Empire. In: The Northern Dynasties: Kingship and Statecraft in Chimor (M. E. Moseley \& A. Cordy-Collins, eds.): 195-226; Washington, D. C.: Dumbarton Oaks Research Library and Collection.

MILLAIRE, J.-F., 2002 - Moche Burial Patterns: An Investigation into Prehispanic Social Structure, 222 pp.; Oxford: Arqueopress.

MILLAIRE, J.-F., 2005 - The sacred character of ruins: Ritual appropriation of Gallinazo architecture in the Virú Valley, Peru. Paper presented at the $70^{\text {th }}$ Annual Meeting of the Society for American Archaeology, 30 March-3 April 2005, Salt Lake City.

MILLAIRE, J.-F., 2010a - Moche Political Expansionism as Viewed From Virú: Recent Archaeological Work in the Close Periphery of a Hegemonic City-State System. In: New Perspectives on Moche Political Organization (J. Quilter \& L. J. Castillo, eds.): 223-251; Washington, D. C.: Dumbarton Oaks Research Library and Collection.

MILLAIRE, J.-F., 2010b - Primary State Formation in the Virú Valley, North Coast of Peru. PNAS, 107 (14): 6186-6191.

MOSELEY, M. E., 1982 - Introduction: Human Exploitation and Organization on the North Andean Coast. In: Chan Chan: Andean Desert City (M. E. Moseley \& K. C. Day, eds.): 1-24; Albuquerque: University of New Mexico Press.

MOSELEY, M. E., 2001 - The Incas and their Ancestors: The Archaeology of Peru, 288 pp.; London: Thames and Hudson.

MujíCA, E. (ed.), 2007 - El Brujo Huaca Cao, centro ceremonial Moche en el valle de Chicama, 339 pp.; Lima: ING Fondos, AFP Integra.

O'NEILL, J. P., 1984 - Introduction: Feather Identification. In: Costumes \& Featherwork of the Lords of Chimor: Textiles from Peru's North Coast (A. P. Rowe): 144-150; Washington, D. C.: The Textile Museum.

PAUL, A., 1982 - The Chronological Relationship of the Linear, Block Color, and Broad Line Styles of Paracas Embroidered Images. In: Pre-Columbian Art History: Selected Readings (A. Cordy-Collins, ed.): 255-277; Palo Alto: Peek Publications.

PAUL, A., 1990 - Paracas Ritual Attire: Symbols of Authority in Ancient Peru, 170 pp.; Norman: University of Oklahoma Press.

PETERS, A. H., 1991 - Ecology and Society in Embroidered Images from the Paracas Necropolis. In: Paracas Art and Architecture (A. Paul, ed.): 240-314; lowa City: University of lowa Press.

PETERS, A. H., 1997 - Paracas, Topará, and Early Nasca: Ethnicity and Society on the South Central Andean Coast; Ithaca: Cornell University. Ph.D. Dissertation. 
PETERS, A. H., 2000 - Funerary Regalia and Institutions of Leadership in Paracas and Topará. Chungará, 32: 245-252.

REIMER P. J., et al., 2004 - IntCal04 terrestrial radiocarbon age calibration, 0-26 cal kyr BP. Radiocarbon, 46: 1029-1058.

ROWE, A. P., 1984 - Costumes \& Featherwork of the Lords of Chimor: Textiles from Peru's North Coast, 190 pp.; Washington, D. C.: The Textile Museum.

ROWE, J. H., 1946 - Inca Culture at the Time of the Spanish Conquest. In: Handbook of South American Indians, vol. 2: The Andean Civilizations (J. H. Steward, ed.): 183330; Washington, D. C.: Bureau of American Ethnology.

ROWE, J. H., 1995 - Behavior and Belief in Ancient Peruvian Mortuary Practice. In: Tombs for the Living: Andean Mortuary Practices (T. D. Dillehay, ed.): 27-41; Washington, D. C.: Dumbarton Oaks Research Library and Collection.

SEILER-BALDINGER, A., 1979 - Classification of Textile Techniques, 114 pp.; Ahmedabad, India: Calico Museum of Textiles.

SQUIER, E. G., 1967 [1869] - A Plain Man's Tomb in Peru. In: Peruvian Archaeology: Selected Readings (J. Rowe \& D. Menzel, eds.): 210-216; Palo Alto: Peek Publications.

STOTHERT, K. E., 1978 - Preparing a Mummy Bundle: Note on a Late Burial from Ancon, Peru. Nawpa Pacha, 16: 13-22.

STOTHERT, K. E., 1979 - Unwrapping an Inca Mummy Bundle. Archaeology, 32: 8-17.

STRONG, W. D. \& EVANS, C., 1952 - Cultural Stratigraphy in the Virú Valley, Northern Peru: The Formative and Florescent Epochs, 373 pp.; New York: Columbia University Press.

TELLO, J. C. \& MEJÍA, T., 1979 - Paracas. Il Parte: Cavernas y Necrópolis, 502 pp.; Lima: Universidad Nacional Mayor de San Marcos.

TOPIC, T., 1990 - Territorial Expansion and the Kingdom of Chimor. In: The Northern Dynasties: Kingship and Statecraft in Chimor (M. E. Moseley \& A. Cordy-Collins, eds.): 177-194; Washington, D. C.: Dumbarton Oaks Research Library and Collection.

UHLE, M., 1899 - Fieldnotes, Catalogue and Correspondence 1899-1900; Berkeley: P. A. Hearst Museum. Manuscript on file.

VÁSQUEZ, V. \& ROSALES, T., 1994 - Estudio zooarqueológico de una muestra de camelidae de Cerro Blanco-valle de Moche; Trujillo: Centro de Investigaciones Arqueobiológicas y Paleoecológicas Andinas. Manuscript on file.

VREELAND, J., 1998 - Mummies of Peru. In: Mummies, Disease \& Cultures (A. Cockburn, E. Cockburn \& Th. A. Reyman, eds.): 154-189; Cambridge: Cambridge University Press.

WILLEY, G. R., 1953 - Prehistoric Settlement Patterns in the Virú Valley, Perú, 453 pp.; Washington, D. C.: Bureau of American Ethnology.

WILLIAMS, J. S., 2005 - Investigating Diet and Dietary Change Using the Stable Isotopes of Carbon and Nitrogen in Mummified Tissues from Puruchuco-Huaquerones, Peru; Calgary: University of Calgary. PhD dissertation.

WOUTER, J. \& ROSARIO-CHIRINOS, N., 1992 - Dye Analysis of Pre-Columbian Peruvian Textiles with High-Performance Liquid Chromatography and Diode-Array Detection. Journal of the American Institute for Conservation, 31: 237-255.

YACOVLEFF, E. \& MUELLE, J. C., 1934 - Un fardo funerario de Paracas. Revista del Museo Nacional, 3: 63-153. 